\title{
Avaliação da relação entre a cobertura vacinal contra o HPV em um estado do Brasil com outros indicadores de saúde
}

\author{
Evaluation of the relationship between vaccination coverage against HPV in a state in \\ Brazil with other health indicators
}
Evaluación de la relación entre la cobertura de vacunación contra el VPH en un estado de Brasil con otros indicadores de salud

Nelmar de Oliveira Mendes ${ }^{1 *}$, Alexsandro Guimarães Reis ${ }^{2}$, Viviane Sousa Ferreira ${ }^{1}$, Ilka Kassandra Pereira Belfort ${ }^{1}$, Arissane de Sousa Falcão ${ }^{1}$, Selma Gomes Samineses ${ }^{3}$, Elis Cabral Victor ${ }^{1}$, Marta de Oliveira Barreiros ${ }^{1}$, Flávia Ferreira Monari ${ }^{1}$, Vanessa Edilene Duarte Martins ${ }^{1}$.

\begin{abstract}
RESUMO
Objetivo: Avaliar a relação entre a situação da vacinação contra o HPV e os indicadores relacionados ao câncer de colo de útero. Métodos: $O$ presente estudo é do tipo descritivo retrospectivo realizado no estado do Maranhão entre 2014 a 2019 com as populações femininas que são alvo da campanha de vacinação. Também foram avaliadas, outras faixas etárias com o objetivo de correlacionar a série histórica para alguns indicadores. Para a tabulação, utilizamos os Sistemas de Informação em Saúde: SIS-PNI, SIH-SUS, IBGE, SISCAN consistentes no site do DATASUS. Resultados: O estado do Maranhão ao longo dos anos, não cumpriu as metas, que englobam vacinar $80 \%$ da população alvo, a situação é pior quando foram observadas a administração da segunda dose da vacina. Essa situação se expressa de forma clara na não modificação da situação dos exames colposcópicos, gastos hospitalares e quantidade de mulheres internadas para realização de procedimentos relacionados ao tratamento radical do câncer de colo de útero. Conclusão: o presente estudo corrobora com os achados de outras pesquisas que mostra que a não efetividade da vacinação traz como resultados a não modificação da situação epidemiológica.
\end{abstract}

Palavras-chave: Papillomaviridae, Avaliação de processos, Sistemas de informação.

\begin{abstract}
Objective: To evaluate the relationship between the situation of HPV vaccination and the indicators related to cervical cancer. Methods: The present study is a retrospective descriptive study carried out in the state of Maranhão from 2014 to 2019 with the female populations that are the target of the vaccination campaign. Populations in other ages were also evaluated in order to correlate the historical series for some indicators. For the tabulation, we use the Health Information Systems: SIS-PNI, SIH-SUS, IBGE, SISCAN consistent on the DATASUS website. Results: The state of Maranhão over the years has not met the targets, which include vaccinating $80 \%$ of the target population, the situation is worse when the administration of the second dose of the vaccine was observed. This situation is clearly expressed in the fact that the status of colposcopic examinations, hospital expenses and the number of women hospitalized for procedures related to the radical treatment of cervical cancer are not changed. Conclusion: the present study corroborates the findings of other studies that show that the ineffectiveness of vaccination results in the non-modification of the epidemiological situation.
\end{abstract}

Keywords: Papillomaviridae, Evaluation of processes, Information systems.

\footnotetext{
${ }^{1}$ Universidade Federal do Maranhão, São Luís - MA. *E-mal: viviane_gnr@hotmail.com

2 Hospital Socorrão I - São Luís - MA.

${ }^{3}$ Centro Especializado de Reabilitação do Olho D’água, São Luís - MA.
} 


\section{RESUMEN}

Objetivo Evaluar la relación entre la situación de vacunación contra el VPH y los indicadores relacionados con el cáncer cervical. Métodos: El presente estudio fue un estudio descriptivo retrospectivo llevado a cabo en el estado de Maranhão de 2014 a 2019 con las poblaciones femeninas que son el objetivo de la campaña de vacunación. También se evaluaron otras edades para correlacionar las series históricas de algunos indicadores. Para la tabulación, utilizamos los Sistemas de Información de Salud: SIS-PNI, SIH-SUS, IBGE, SISCAN, consistentes en el sitio web de DATASUS. Resultados: El estado de Maranhão a lo largo de los años no ha cumplido los objetivos, que incluyen la vacunación del $80 \%$ de la población objetivo, la situación es peor cuando se observó la administración de la segunda dosis de la vacuna. Esta situación se expresa claramente en el hecho de que el estado de los exámenes colposcópicos, los gastos hospitalarios y el número de mujeres hospitalizadas por procedimientos relacionados con el tratamiento radical del cáncer cervical no cambian. Conclusión: el presente estudio corrobora los hallazgos de otros estudios que muestran que la ineficacia de la vacuna resulta en la no modificación de la situación epidemiológica.

Palabra clave: Papillomaviridae, Evaluación de procesos, Sistemas de información.

\section{INTRODUÇÃO}

O Papilomavírus Humano (HPV), é um grupo de mais de 150 tipos virais que infecta as células epiteliais basais cutâneas ou mucosas e é altamente prevalente na população sexualmente ativa. O HPV é assintomático e é classificado de acordo com o risco de desenvolver o câncer. Os sub-tipos de alto risco mais comuns são os tipos 16 e 18 e estão associados ao carcinoma de vagina, ânus e colo de útero; os de baixo risco, predominantemente os tipos 6 e 11, causam desenvolvimento de verrugas acuminados (LETO MGP, et al., 2011; NAKAGAWA JTT, et al., 2010; GIRIANELLI VR, et al., 2010).

Em relação ao câncer de colo de útero os tipos 16 e 18 são os mais perigosos, estão presentes em cerca de $70 \%$ dos casos de cânceres de colo de útero. Entretanto o tipo 16 é considerado o mais fatal, responsável por $50 \%$ dos casos de canceres de colo de útero em todo o mundo (BRASIL, 2018).

Os tipos 6 e 11 estão associados a 90\% da ocorrência de verrugas na região oral e genital. O Brasil tem similaridade no perfil de prevalência da doença com o restante do mundo $53,2 \%$ para o HPV 16 e 15,8\% para o HPV 18. O câncer do colo do útero é uma doença preocupante e que pode levar a óbito. A sua alta incidência e altas taxas de mortalidade justificam a grande preocupação mundial acerca do tema (WHO, 2016).

Com o objetivo de prevenir a infecção e reduzir a incidência de casos de câncer de colo uterino, foi criada a vacina anti-HPV, o qual há dois tipos que são comercializadas no Brasil: a quadrivalente e a bivalente. Até onde se sabe, a duração da proteção da vacina é de mais de 5 anos após esquema vacinal completo, quando a vacina quadrivalente foi implantada pelo Programa Nacional de Imunizações (PNI), era necessária a administração de três doses, havendo posteriormente, sido modificado o esquema vacinal para duas doses (ZARDO MRI, et al., 2014; RUAS BRB, et al., 2017).

Segundo dados do Instituto Nacional do Câncer (INCA), são esperados 17,11 casos de câncer de colo de útero para cada mil mulheres no Brasil no ano de 2014. Devido o conhecimento prévio da população, qualitativamente incompleto e por vezes equivocado, a adesão à vacinação se torna insuficiente para atingir a meta de prevenção. Enquanto o Brasil não avança na imunização, países como a Austrália vão se destacando na erradicação quase completa do HPV, reduzindo ainda mais as complicações causadas pela doença viral cujo sucesso consiste na associação dos programas com o ambiente escolar (PEREIRA RGV, et al., 2016; MOTA CV, 2018).

Para que o programa de vacinação contra o HPV seja eficaz é necessário que a população seja devidamente informada sobre a enfermidade. Os meios de informação são diversos, com a criação das equipes de saúde da família, a figura do agente comunitário de saúde passa a ser fundamental nesse trabalho, existem muitas barreiras para a aceitação da vacinação e uma delas é o pensamento dos pais de 
que a imunização possa ser um incentivo à uma vida sexual precoce e promíscua, podendo levar ao número de parceiros sexuais e prática de sexo sem proteção. A questão com a segurança da vacina e dúvidas quanto à eficácia também é um fator de muita influência dos pais aos filhos para não se vacinarem (SOUSA PDL, et al., 2018; GRANDAHL M, et al., 2017; ALBRIGHT K, et al., 2017).

A escolha pela faixa etária de 9 a 14 anos pelo Ministério da Saúde para a população alvo para a vacinação contra o HPV, se deve primeiramente a costumes relacionados ao povo brasileiro, nessa idade os pais ainda tem o costume de levar seus filhos para vacinar e no geral, nessa faixa etária as crianças e adolescentes ainda não iniciaram a sua vida sexual. Uma justificativa mais fisiológica e imunológica é que nesse período a vacinação proporciona níveis mais altos de anticorpos se comparado à imunidade natural produzida pela infecção do HPV (BRASIL, 2018).

As políticas de saúde no Brasil, são fundamentados no cuidado primário em saúde, o Programa de Saúde da Família estabelece que a saúde da mulher é uma das prioridades e o cuidado da equipe principalmente da enfermagem garante o cumprimento das metas de realização de exames citopatológicos e vacinação contra o HPV, com toda essa estrutura voltada às ações a favor da saúde da mulher as expectativas são que haja uma boa promoção e prevenção dos cânceres de colo de útero (CARNEIRO CPF, et al., 2019).

O Ministério da Saúde investiu o equivalente a $R \$ 43,01$ por dose de vacina, o objetivo era que com esse investimento, em pouco tempo o Brasil pudesse contar com diminuições drásticas na quantidade de casos esperados por ano, atualmente quase 2 bilhões de reais são gastos no Brasil com tratamento de canceres, se considerarmos que o câncer de colo de útero é um dos mais prevalentes, uma campanha bem-sucedida acerca da vacinação contra o HPV geraria um bom resultado na dispensação de despesas públicas, visto que este está relacionado a $90 \%$ dos casos de câncer de colo de útero (BRASIL, 2018).

O objetivo do presente estudo foi realizar uma correlação entre a situação da vacinação contra o HPV e os indicadores relacionados a ocorrência do câncer de colo de útero e outros indicadores de qualidade relacionados à utilização de serviços hospitalares por esse público nos procedimentos relacionados a esse agravo avaliando séries temporais.

\section{MÉTODOS}

\section{Local, população e desenho do estudo}

Foi realizado um estudo epidemiológico descritivo retrospectivo de abordagem quantitativa. Os participantes do estudo foram principalmente meninas na faixa etária alvo para a vacinação contra o HPV (09 a 14 anos), outras faixas etárias também foram consideradas apenas a nível de comparação com a população alvo, todas residentes do estado do Maranhão. O estudo foi feito com recorte temporal do ano de 2014 a 2019, todas as informações estão presentes no DATASUS nos Sistemas: Sistema de Informação do Instituto Brasileiro de Geografia e Estatística (IBGE), Sistema de Informação em Saúde do Programa Nacional de Imunização (SIS-PNI) e Sistema de Informação Hospitalar do SUS (SIH-SUS) e Sistema de Informação de Câncer de Colo te útero e mama (SISCAN).

\section{Variáveis analisadas}

Foram utilizadas como base para avaliação parâmetros de qualidade e informações que ao longos dos anos de 2014 a 2019 representassem a capacidade do estado do Maranhão em promover um ambiente saudável às mulheres e que pudessem nos dar um retrato da situação atual do câncer do colo de útero e perspectivas em relação aos gastos com os principais procedimentos relacionados a essa doença, entendendo que a infecção por HPV é um dos grandes causadores disso, o período de 2008 a 2013 foi considerado em uma tabulação para comparação do período principal estabelecido para avaliação nesse estudo.

\section{Os indicadores utilizados para avaliação foram}

- Relação entre a quantidade de meninas residentes no estado do Maranhão na faixa etária de 09 a 14 anos e a administração da primeira e segunda dose da vacina entre os anos de 2014 e 2019. Para a 
elaboração desse indicador foi utilizado o Sistema de Informação do Programa Nacional de Imunização (SIS$\mathrm{PNI}$ ) e dados demográficos do IBGE;

- Relação entre a presença de epitélio metastático na faixa etária de 09 anos, 10 a 14 anos e de 15 a 19 anos em pessoas do sexo feminino e residentes no estado do Maranhão e sua relação com a administração da primeira e da segunda dose da vacina entre os anos de 2014 e 2019. Para a elaboração desse indicador foi usada como base os Sistemas de Informação Hospitalar (SIH-SUS) e dados demográficos do IBGE;

- Presença de epitélio metastásico por faixa etária 9 anos, 10 a 14 anos, 15 a 19 anos dos anos de 2014 a 2019 na população residente do estado do Maranhão. As tabulações utilizaram como base o Sistema de Informação do Câncer de Mama e Útero (SISCAN) e dados demográficos do IBGE;

- Comparação entre as proporções das séries históricas de 2008 a 2013 e 2014 a 2019 de neoplasias e carcinomas em mulheres internada por faixa etária por 10.000 mil habitantes no estado do Maranhão. Para a realização das tabulações utilizamos as bases de dados do SIH-SUS;

- Média de gastos ao longo dos anos de 2014 a 2019 com os procedimentos: Histerectomia com anexectomia (uni e bilateral) em oncologia, Histerectomia com ressecção de órgãos contíguos em oncologia; Histerectomia total ampliada em oncologia; Histerectomia total em oncologia de residentes do Maranhão. Todos os procedimentos elencados para a tabulação estão presentes no Sistema de Gerenciamento da Tabela de Procedimentos, Medicamentos, Órteses e Próteses do Sistema Único de Saúde (SIGTAP), registrado sob os números de procedimento, consecutivamente: 04160600-48, 04160600-56, 04160600-64 e 04160600-72;

- Quantidades de Autorizações de Internações Hospitalares (AlH's) aprovadas ao longos dos anos de 2014 a 2019 no procedimento Amputação Cônica de colo de útero com colpectomia em Oncologia e valores gastos com o procedimento na população de mulheres em geral, o procedimento está presente no Sistema de Gerenciamento da Tabela de Procedimentos, Medicamentos, Órteses e Próteses do Sistema Único de Saúde (SIGTAP) sob o código de procedimento 04160600-13, para a tabulação utilizamos como base o Sistema de Informação Hospitalar (SIH-SUS).

O período da busca de informações e tabulações abrangeu o período de 01/10/2019 a 14/02/2020. Utilizamos os tabuladores: Tabnet, Microsoft Excel, Numbers for Macbook para construção das tabelas e Gráficos. Os dados foram analisados e organizados em forma de porcentagens, médias e proporções.

\section{RESULTADOS E DISCUSSÃO}

\section{Meta de vacinação e relação entre primeira e segunda dose}

No Brasil, adotou-se a vacina quadrivalente que consiste em duas doses aplicadas com intervalo de três a seis meses. A cobertura vacinal satisfatória é um fator fundamental para a erradicação do câncer de colo de útero. O início das campanhas de vacinação aconteceu mais tarde, porém como mostram os resultados do presente estudo no estado do Maranhão não houve o cumprimento das metas estabelecidas, isso nos leva a crer que os resultados alcançados em outros países não serão a nossa realidade nos próximos anos (CAVALCANTI CD, et al., 2019; BRALSFORD KJ e JAMIRSON, 2019).

Mundialmente muitas manifestações foram realizadas contra a vacinação do HPV, pois muitos acreditavam que a vacina serviria de um estímulo para iniciação da vida sexual. Bispos católicos em várias cidades se opuseram de forma veemente à vacinação contra o HPV porque acreditavam que a abstinência era a única opção saudável de prevenir o câncer cervical, esse pode ser um dos motivos que justifiquem os resultados da Gráfico 1 do presente estudo, considerando que mais da $80 \%$ da população brasileira é cristã (GUINCHON JR, et al., 2013).

As pesquisas mostram que a vacinação contra o HPV não está associada ao aumento do comportamento sexual de risco em meninas e adolescentes. A abordagem da população alvo é a vacinação, uma avaliação realizada em uma escola em um município do estado do Tocantins revelou que dos 119 cartões de vacina 
avaliados 47,05\% encontravam-se com atraso da vacina contra o HPV, esses resultados corroboram com os resultados do presente estudo que expõem a situação grave da não vacinação de populações alvo (DONKEN R, et al., 2018; SINKA K, et al., 2014; FEITOSA LG, et al., 2019; GONÇALVES LA, et al, 2018).

A população de meninas e adolescentes na faixa etária estabelecida para vacinação no estado do Maranhão é de 883.386 pessoas. As metas do Ministério da Saúde revelam que $80 \%$ da população alvo deveria ser vacinada com a primeira e segunda dose pois somente assim, seria garantido a imunização. Os resultados encontrados no Gráfico 1 mostram que no ano de 2014 (ano em que a vacinação contra o HPV foi incluída no calendário vacinal) 208.214 meninas receberam a primeira dose da vacina, porém somente 129.345 receberam a segunda dose da vacina.

Os números decrescem ao longo dos anos, se considerarmos a segunda dose no ano de 2015 somente 97.653 pacientes foram imunizadas, 44.208 em 2016, o ano de 2017 e 2018 são os anos em que o número de primeiras e segundas doses mais se aproximam, representando boa busca ativa porém, bastante distante das metas estipuladas ( 77.350 de primeira dose e 57.550 de segunda dose em 2017 e 49.962 de primeira dose e 45.969 de segunda dose em 2018). O ano de 2019 está representado com os piores resultados 26.562 meninas receberam a primeira dose e 24.588 receberam a segunda dose.

No total 399.313 meninas foram imunizadas com a segunda dose da vacina, isso representa apenas $45,20 \%$ da população alvo, outro fator agravante é que esse número foi atingido ao longo de 6 anos de intervenção, considerando que a imunização é garantida somente por 5 anos, os achados mostram que as equipes de saúde precisam melhorar o seu trabalho para garantir a imunização das populações alvo.

Gráfico 1 - Relação entre a quantidade de meninas residentes no estado do Maranhão na faixa etária de 09 a 14 anos e a administração da primeira e da segunda dose da vacina entre os anos de 2014 e 2019

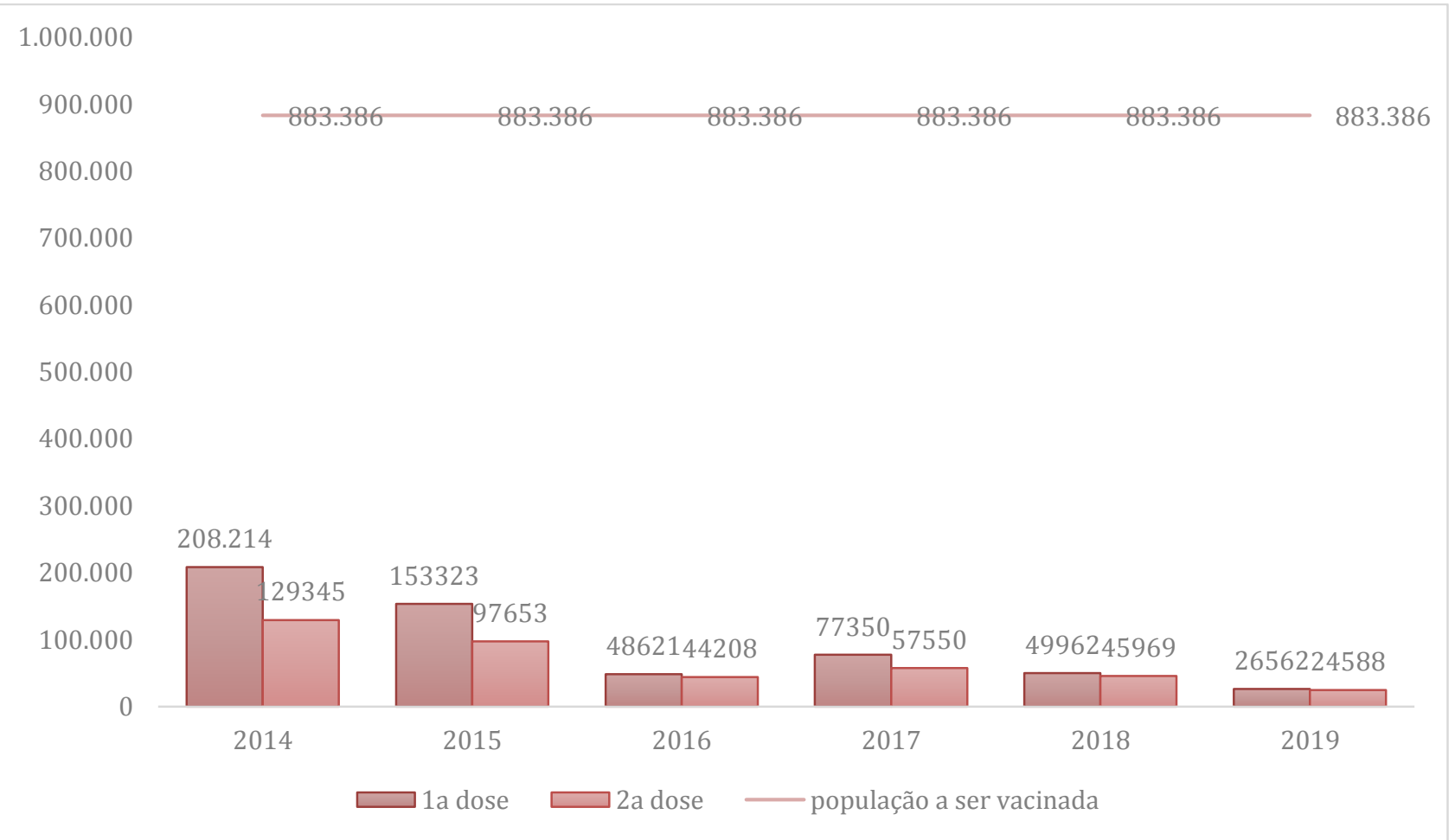

Fonte: DATASUS, 2020.

\section{Relação entre epitélio metastásico no resultado da colposcopia e imunização completa}

$O$ item que mais influencia os resultados da colposcopia é a presença do HPV, o protocolo de rastreamento do câncer de colo de útero passou a ser de 5 em 5 anos nos EUA, no Brasil o protocolo é a realização dos exames anualmente. Pesquisas demonstram que um programa de vacinação de rotina em adolescentes de 
12 e 13 anos de idade está associada a uma profunda redução da doença cervical sete anos depois do seu início. Os resultados demonstram de forma clara que a vacinação oportuna é uma medida confiável da avaliação do risco de câncer em mulheres (NAM K, 2018; PALMER T, et al., 2019). Nos resultados obtidos no presente estudo, relacionamos a presença de epitélio metastásico com a situação vacinal das mulheres e pudemos comprovar que o não cumprimento das metas estipuladas contribuiu para a manutenção e até aumento de resultados negativos na colposcopia.

Na Austrália, se houver uma manutenção nas campanhas de vacinação a incidência anual de câncer do colo do útero pode diminuir para somente um novo caso a cada 100.000 mulheres na faixa etária de risco no ano de 2066 porém, se o programa de triagem for descontinuado, a incidência anual será de aproximadamente três casos por 100.000 mulheres (HALL MT, et al., 2019). Os resultados de um estudo de base populacional indicam uma redução no número absoluto de mulheres jovens encaminhadas à colposcopia quando receberam imunização completa contra o HPV. Na Escócia evidenciou-se que existe uma redução significativa na prevalência de HPV 16/18 e NIC 2 em mulheres de 20 a 25 anos de idade que fizeram colposcopia, existe uma grande dificuldade em cumprir as metas de vacinação na população feminina como visto na tabela anterior, por isso a presença de alterações histológicas nos tecidos frequente (CRUICKSHANK ME, et al., 2017; MUNRO A, et al., 2017; ANIL K, et al., 2017).

O Gráfico 2 representa a presença de epitélio metastásico na população de meninas de 09 a 19 anos ao longo dos anos de 2014 a 2019. A expectativa é que com o passar dos anos a quantidade de meninas na faixa etária de risco diminuísse com a inserção da vacina de HPV no calendário de vacinação, situação que não é observada no Gráfico 2 a supra citado.

Foram inseridas na tabulação de "presença de epitélio metastásico" além da população de 09 a 14 nos, adolescentes de 15 a 19 anos porque a população que tinha de 09 a 14 anos em 2014 atualmente está na faixa etária de 15 a 19 anos de idade. Os anos de 2014 e 2015 representam as menores ocorrências de aparecimento de epitélio metastásico de toda a série histórica ( 2014 - 869 casos e 2015 - 865 casos). Apesar da vacinação iniciada em 2014, a partir do ano de 2016 o Gráfico 2 demonstra uma tendência forte de crescimento da anormalidade histológica revelando 1.200 casos em 2016, 1.458 em 2017, 1.652 casos em 2018 e 1.769 casos em 2019 o que representa forte ligação de alterações crescentes aos exames colposcópicos com a insuficiência na vacinação completa em tempo oportuno.

Gráfico 2 - Relação entre a presença de epitélio metastático na faixa etária de 09 anos, 10 a 14 anos e de 15 a 19 anos em pessoas do sexo feminino e residentes no estado do Maranhão e sua relação com a administração da primeira e da segunda dose da vacina entre os anos de 2014 e 2019

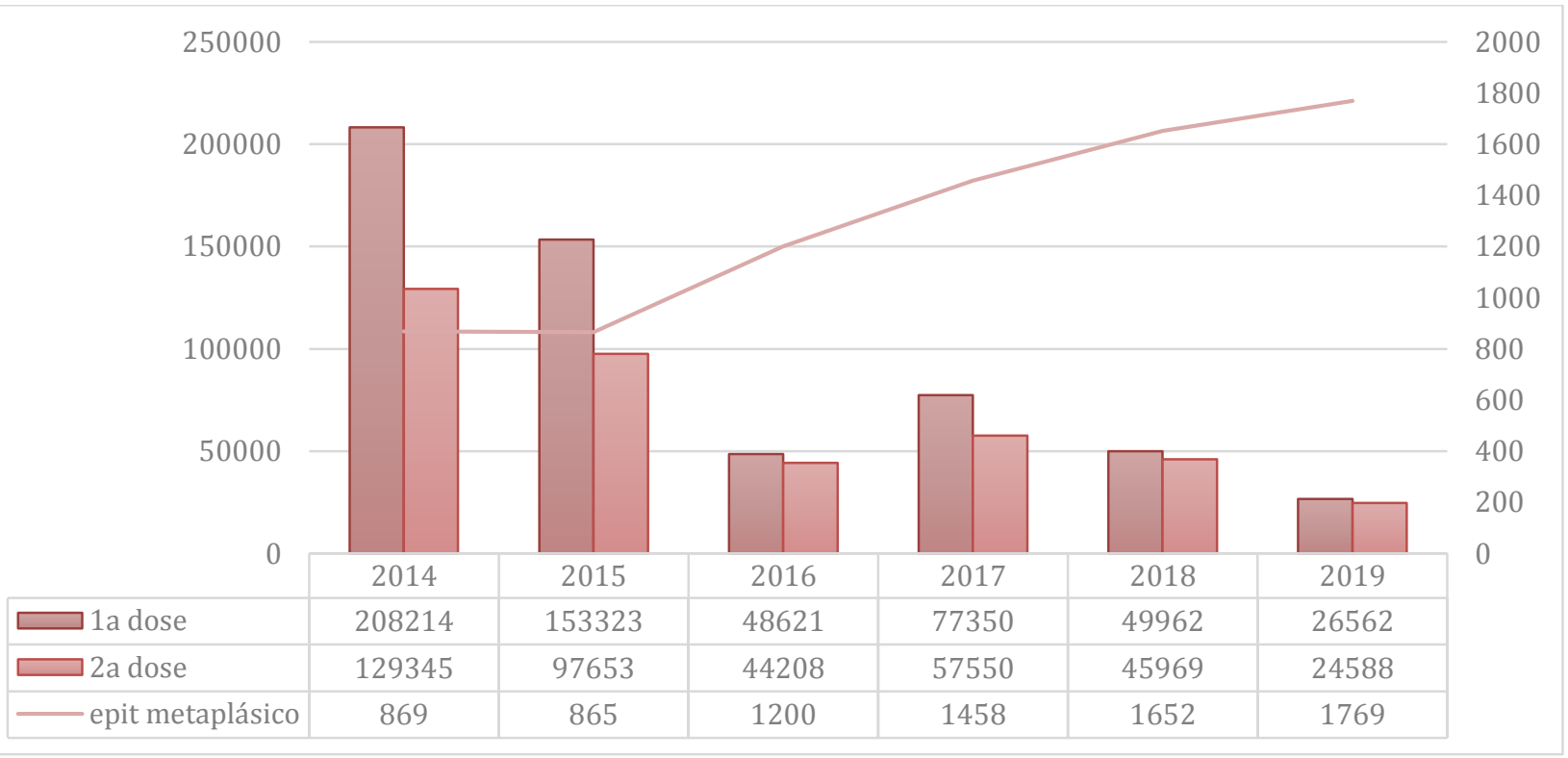

Fonte: DATASUS, 2020 
Apesar da vacina contra o HPV ter como objetivo a diminuição na incidência de novos casos de HPV e consequentemente cânceres, as pesquisas mostram que ela pode funcionar como um tratamento para aqueles que já contraíram o HPV eliminando verrugas por exemplo. Pesquisadores comprovaram que tanto na vacinação preventiva como terapêutica a vacinação contra o HPV apresentou vantagens mesmo em uma infecção recorrente $(\mathrm{CHOI} \mathrm{H,} \mathrm{2019).}$

Em um estudo realizado no estado do Maranhão no ano de 2011 foi demonstrado que 12,5\% de todos os exames realizados apresentaram epitélio metaplásico. Somente $18,9 \%$ dos das mulheres que apresentaram alterações metaplásicas estão na faixa etária de menores de 25 anos de idade (SILVA DSM, et al., 2014).

A Tabela 1 revela a frequência dos epitélios metastásicos por faixas etárias. Em todos os anos o grupo de 15 a 19 anos apresentou os maiores números e de forma crescente ao longo dos anos, 834 em 2014 e 1.707 em 2019, representando um aumento de mais de $100 \%$ ao longo dos anos.

A menor incidência foi no grupo de meninas de até 9 anos, possivelmente porque a realização de exames no grupo é menor, os resultados dessa pesquisa mostram a necessidade de um cuidado especial à essas pacientes especialmente na faixa etária de 15 a 19 anos onde se observou um aumento de mais de $100 \%$ de alteração em 6 anos.

Tabela 1 - Presença de epitélio metastásico por faixa etária 9 anos, 10 a 14 anos, 15 a 19 anos dos anos de 2014 a 2019 na população residente do estado do Maranhão.

$\begin{array}{llllllll}\text { Faixa etária } & 2014 & 2015 & 2016 & 2017 & 2018 & 2019 & \text { Total }\end{array}$

$\begin{array}{llllllll}\text { Total } & 869 & 865 & 1.200 & 1.458 & 1.652 & 1.769 & 7.813\end{array}$

$\begin{array}{lccccccc}\text { Até } 9 \text { anos } & 5 & 7 & 10 & 5 & 11 & 4 & 42 \\ \text { Entre } 10 \text { a } 14 \text { anos } & 30 & 42 & 38 & 48 & 57 & 58 & 273 \\ \text { Entre } 15 \text { a } 19 \text { anos } & 834 & 816 & 1.152 & 1.405 & 1.584 & 1.707 & 7.498\end{array}$

Fonte: DATASUS, 2020.

O Gráfico 3 compara duas séries temporais em proporções com o objetivo de avaliar o risco que a mulher em cada uma das faixas etárias tem de adoecer por carcinoma e neoplasia de colo de útero no estado do Maranhão. Em todas as faixas etárias avaliadas o risco de desenvolver a doença é maior do período de 2014 a 2019. A faixa etária de 60 a 69 anos é a que apresenta a maior disparidade, o risco atual em comparação ao período de 2008 a 2013 é de quase 7 vezes mais. 
Gráfico 3 - Comparação entre as proporções das séries históricas de 2008 a 2013 e 2014 a 2019 de neoplasias e carcinomas em mulheres internada por faixa etária por 10.000 mil habitantes no estado do Maranhão.

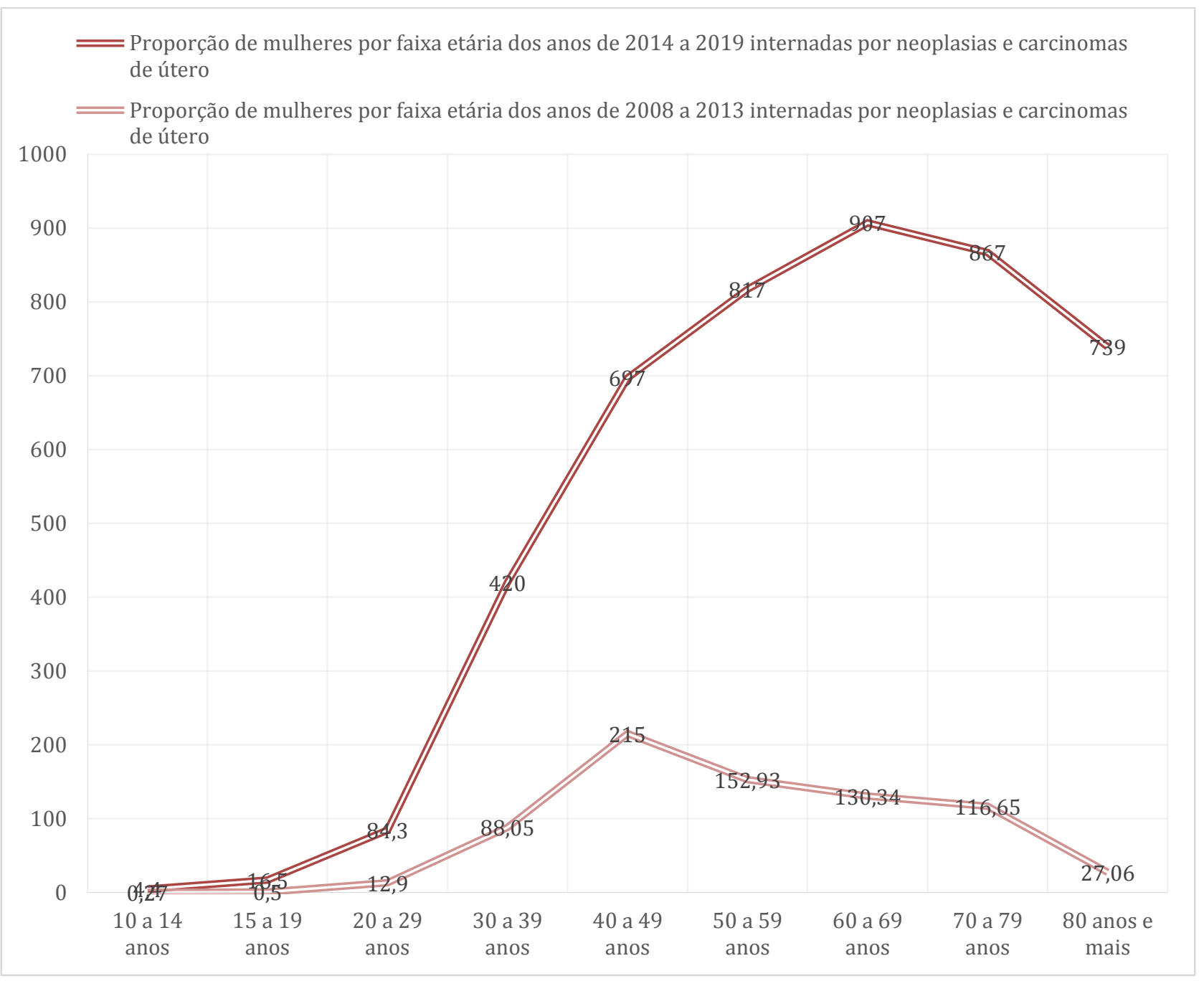

Fonte: DATASUS, 2020

\section{Série histórica de gastos hospitalares relacionados ao câncer de colo de útero ao longo dos anos}

Com a imunização bem-sucedida, a vacina quadrivalente pode reduzir o número de exames de colposcopias e biópsias e a quantidade de tratamentos de lesões precursoras do câncer de colo do útero. Um dos pontos mais relevantes das políticas públicas de saúde é mensurar a eficiência econômica das mesmas em se tratando de custos e benefícios (SANCHES EB, 2010; ZAHDI MR, 2010).

Não foi um objetivo desse estudo traçar o perfil sócioeconômico das pacientes internadas com complicações relacionadas ao câncer de colo de útero, porém estudos com essa abordagem auxiliam a melhorar a situação encontrada no Gráfico 4.

A tabulação do Gráfico 4 revela a relação aos gastos hospitalares relacionados a procedimentos gerados a partir dos cânceres de colo de útero. Observou-se que o ano de 2017 teve o maior gasto $(R \$ 6.594,34$ por paciente).

O aumento de gastos hospitalares por paciente é um importante indicador porque a mesma Autorização de Internação Hospitalar pode ser faturada com o valor mais alto ou mais baixo representando assim que quando há necessidade da paciente ficar mais tempo internada, mais procedimentos complexos, portanto, 0 aumento na média representa maiores despesas individuais o que revela um ponto negativo. 
Gráfico 4 - Média de gastos ao longo dos anos de 2014 a 2019 com os procedimentos: Histerectomia com anexectomia (uni e bilateral) em oncologia, Histerectomia com ressecção de órgãos contíguos em oncologia; Histerectomia total ampliada em oncologia; Histerectomia total em oncologia de residentes do Maranhão.

\section{média de gastos por paciente ( em $\mathrm{R} \$$ )}

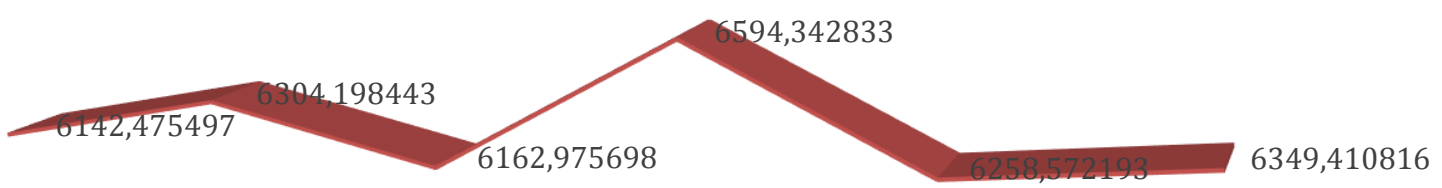

2014

2015

2016

2017

2018

2019

média de gastos por paciente

Fonte: DATASUS, 2020.

Quando um serviço de imunização é efetivo a expectativa é que os casos de cânceres de colo de útero e gastos hospitalares atrelados a ele praticamente desapareçam, o que se observa no presente estudo é que acontecem aumentos de forma progressiva.

No Brasil os canceres de colo de útero continuam sendo um grande desafio para o setor saúde, se considerarmos que as mulheres também participam de forma efetiva e as vezes exclusiva da renda do lar isso afeta não somente o sistema público de saúde mas a economia das famílias (HALL MT, et al., 2019; SANTOS RS e MELO ECP, 2010). A Tabela 2 representa a média de AlHs, AlHs aprovadas e valor total do procedimento amputação cônica de colo de útero em oncologia com colpectomia. Não foram observadas muitas diferenças em relação ao valor médio de AlH ao longo dos anos.

A quantidade de pacientes internados ao longo dos anos apresentou variação em relação à quantidade de pacientes que fizeram o procedimento, o ano de 2019 foi o ano que mais houve internações 51 pacientes, seguido do ano de 2018 (27 pacientes). O procedimento é realizado quando há a necessidade de um procedimento mais sério quando identificado a necessidade de uma intervenção mais radical em relação ao descontrole do epitélio metastásico.

Tabela 2 - Quantidades de Autorizações de Internações Hospitalares (AlH's) aprovadas ao longo dos anos de 2014 a 2019 no procedimento Amputação Cônica de colo de útero com colpectomia em Oncologia e valores gastos com o procedimento na população de mulheres em geral.

\begin{tabular}{cccc}
\hline Ano processamento & Média AlH & AlHs aprovadas & Valor total \\
\hline 2014 & $1.876,96$ & 18 & $33.785,37$ \\
\hline 2015 & $1.942,49$ & 7 & $13.596,83$ \\
\hline 2016 & $1.902,69$ & 4 & $7.610,76$ \\
\hline 2017 & $2.202,42$ & 1 & $2.202,42$ \\
\hline 2018 & $1.843,78$ & 27 & $49.782,09$ \\
\hline 2019 & $1.914,24$ & 51 & $97.626,58$ \\
\hline
\end{tabular}

Fonte: DATASUS, 2020. 


\section{CONSIDERAÇÕES FINAIS}

As intervenções do Ministério da Saúde no sentido de promover a vacinação contra o HPV e políticas voltadas à saúde da mulher são de fundamental importância para reduzir os índices de câncer de colo de útero. O presente estudo corrobora com os achados de outras pesquisas que mostra que a falência nas estratégias de vacinação e de promoção e prevenção não modifica situações epidemiológicas, gastos hospitalares e resultados desfavoráveis de exames laboratoriais. O estado do Maranhão frente a esses resultados, necessita de uma intervenção urgente de forma que no futuro a situação seja modificada e as mulheres possam ter uma perspectiva melhor em relação à doença.

\section{REFERÊNCIAS}

1. ALBRIGHT K, et al. Noninitiation and Noncompletion of HPV Vaccine Among English-and Spanish-Speaking Parents of Adolescent Girls: A Qualitative Study. Academic Pediatrics. 2017;17(7):778-784.

2. ANIL K, et al. Effect of prophylactic Human Papillomavirus (HPV) vaccination on oral HPV infections among young adults in the United States. Journal of Clinic Oncology. 2017; 36: 262-267.

3. BRALSFORD KJ, JAMIRSON E. Following Australia's lead to eradicate cervical cancer. BMJ. 2019; 36(6): 49-55.

4. BRASIL, MINISTÉRIO DA SAÚDE. Informe técnico da ampliação da oferta das vacinas papilomavírus humano 6,11 , 16 e 18 (recombinante) -vacina HPV quadrivalente emeningocócica C (conjugada), Brasilia 2018.

5. BROWER AF, et al. HPV vaccination has not increased sexual activity or accelerated sexual debut in a college-aged cohort of men and women. BMC Public Health. 2019; 25(19): 821- 825.

6. CARNEIRO CPF, et al. O papel do enfermeiro frente ao câncer de colo uterino. Revista Acervo Saúde. 2019 ; 35 (25): $1-9$.

7. CAVALCANTI CD, et al. Eficácia da dose única na imunização contra o HPV. Brazilian Journal of Surgery and Clinical Research. 2019; 28(1):61-70.

8. $\mathrm{CHOI} \mathrm{H}$. Can quadrivalent human papillomavirus prophylactic vaccine be an effective alternative for the therapeutic management of genital warts? An exploratory study. International Brazilian Journal of Urology. $2019 ; 45$ (2): $361-368$.

9. CRUICKSHANK ME, et al. Reduction in colposcopy workload and associated clinical activity following human papillomavirus (HPV) catch-up vaccination programme in Scotland: an ecological study. BJOG. 2017; 1-8.

10. DEPARTAMENTO DE INFORMÁTICA DO SUS (DATASUS). Informações de Saúde, Epidemiológicas e Morbidade, Vacinação e gastos hospitalares. Disponível em:<http://www.datasus.gov.br/. Acesso em: 14 out. 2019.

11. DONKEN R, et al. Effect of human papillomavirus vaccination on sexual behaviour among young females. Le Médecin de famille canadien. 2018; 64: 509-513.

12. FEITOSA LG, et al. Imunização contra papilomavirus humano em escolas municipais: relato de experiência. Revista de Enfermagem da UFPE. 2019; 13(24): 12-18.

13. FONSECA AJ, et al. Epidemiologia e impacto econômico do câncer de colo de útero no Estado de Roraima: a perspectiva do SUS. Revista Brasileira de Ginecologia e Obstetetrícia. 2010; 32(8): 386-392.

14. GIRIANELLI VR, et al. Prevalência de HPV em mulheres assistidas pela estratégia saúde da família na Baixa Fluminense do Estado do Rio de Janeiro. Revista Brasileira de Ginecologia e Obstetetrícia, 2010; 32(1): 39-46.

15. GONÇALVES LA, et al. Avaliação da cobertura vacional contra o papiloma vírus humano a partir da vacina HPV quadrivalente recombinante nos municípios do 9ํㅡúcleo regional de saúde da Bahia. Saúde n Revista Mosaicum. 2018; 27:147-158.

16. GRANDAHL M, et al. To consent or decline HPV Vaccination: A Pilot Study at the Start of the National School-Based Vaccination Program in Sweden. Journal of School Health. 2017;87(1):62-70.

17. GUINCHON JR, et al. Citizen intervention in a religious ban on in-school HPV vaccine administration in Calgary, Canada. Preventive Medicine. 2013; 57(5):409- 413.

18. HALL MT, et al. The projected timeframe until cervical cancer elimination in Australia: a modelling study. Lancet Public Health. 2019; 4:19-27.

19. LETO MGP, et al. Infecção pelo Papilomavírus Humano: Etiopatogenia, Biologia Molecular e Manifestações Clínicas. Anais Brasileiro de Dermatologia, 2011; 86(2): 306-317.

20. MOTA CV. HPV: Por que a vacinação de adolescentes contra Vírus de transmissão sexual que causa câncer não avança no Brasil?. BBC Brasil, 2018. Disponível em: https://www.bbc.com/portuguese/brasil-44705298. Acesso em: 10 de nov. 2019.

21. MUNRO A, et al. The impact of human papillomavirus type on colposcopy performance in women offered HPV immunisation in a catch-up vaccine programme: a two-centre observational study. BJOG. 2017;124: 1394-1401.

22. NAKAGAWA JTT, et al. Vírus HPV e Câncer de Colo de Útero. Revista Brasileira de Enfermagem, 2010; 63(2): 307311.

23. NAM K. Colposcopy at a turning point. Obstetrics \& Gynecology Science. 2018;61(1):1-6.

24. PALMER T, et al. Prevalence of cervical disease at age 20 after immunisation with bivalent HPV vaccine at age 12 13 in Scotland: retrospective population study. BMJ. 2019; 36(5):1-10.

25. PEREIRA RGV, et al. A Influência do conhecimento na atitude frente à vacina contra o Papilomavírus Humano: ensaio clínico randomizado. ABCS Health Science, 2016; 41(2): 78-83. 
26. RUAS BRB, et al. Estratégia e adesão da vacinação contra HPV no município de Amparo, São Paulo, Brasil. Saúde em Foco, 2017; 1(2); 61-71.

27. SANCHES EB. Prevenção do HPV: A utilização da Vacina nos serviços de saúde. Revista Saúde e Pesquisa. 2010; 3(2): 255-261.

28. SANTOS RS, MELO ECP. Internação por câncer de mama e colo de útero no Brasil. Revista de Pesquisa Cuidado é Fundamental. 2010;2(1):217-219.

29. SILVA DSM, et al. Rastreamento do câncer do colo do útero no Estado do Maranhão, Brasil. Ciência \& Saúde Coletiva. 2014; 19(4):1163-1170.

30. SINKA K, et al. Achieving high and equitable coverage of adolescent HPV vaccine in Scotland. Journal of Epidemiology and Community Health. 2014; 68:57-63.

31. SOUSA PDL, et al. Conhecimento e aceitabilidade da vacina para o HPV entre adolescentes, pais e profissionais de saúde: elaboração de constructo para coleta e composição de banco de dados. Joural of Human Growth Development, 2018; 28(1):58-68.

32. WHO, World Health Organization. Weekly epidemiological record. Relevé épidémiologique hebdomadaire. Weekly epidemiological record, $n^{\circ} .43,24$ october 2014.

33. ZAHDI MR, et al. Hepatite A: avaliação do custo-benefício da prevenção pela vacina - Paraná, Brasil. Revista Brasileira de Medicina de Família e Comunidade. 2010; 4(16):281-288.

34. ZARDO GP, et al. Vacina como agente de Imunização do HPV. Ciência e Saúde Coletiva, 2014; 19(9):3799-3808. 